\title{
Graphene filled polymer nanocomposites
}

\author{
Raquel Verdejo,* M. Mar Bernal, Laura J. Romasanta, and Miguel A. Lopez-Manchado
}

\begin{abstract}
${ }_{5}$ Graphene has attracted the attention of a growing number of scientists from several disciplines due to its remarkable physical properties and chemical functionalisation capabilities. This review presents an overview of graphene/polymer nanocomposites discussing preparation, properties and potential applications. The challenges and outlook of these emerging polymer nanocomposites are also discussed.
\end{abstract}

\section{Introduction}

Industrial and academic research on polymer nanocomposites was pursued to provide added value properties to the neat polymer, without sacrificing its processability or adding 15 excessive weight. ${ }^{1}$ In this context, carbon-based nanoparticles, in particular carbon nanotubes (CNTs), offered the potential to combine several properties, such as mechanical strength, electrical conductivity and thermal stability, among others. This potential arises from the remarkable properties of the 20 nanotubes, which are based on the building block of all graphitic allotropes: graphene. Although significant advances have been made in the use of carbon nanotubes as reinforcements of polymer matrices, there are still unresolved issues such as the tendency of nanotubes to agglomerate 25 during processing, the limited availability of high-quality nanotubes in large quantities and the high cost of their production. Hence, graphene sheets provide an alternative option to produce functional nanocomposites due to their excellent properties and the natural abundance of its 30 precursor, graphite. $^{2}$

Graphene is a two-dimensional, one-atom-thick carbon sheet with a planar honeycomb lattice. Defect-free graphene presents outstanding physical properties, such as high intrinsic mobility and ballistic transport, high thermal conductivity and 35 Young's modulus, an optical transmittance of almost $98 \%$ and large specific surface area. ${ }^{3-5}$ The most widely used methods to synthesise this high quality, defect-free graphene sheets have been micromechanical cleavage of graphite ("Scotch tape" or peel off method), ${ }^{6}$ and chemical vapour deposition ${ }_{40}(\mathrm{CVD})^{7,8}$ (Fig 1, a-e). However, their production yield is relatively small and, in the case of the micromechanical cleavage, time consuming which hinder the effective and fullexploitation of these materials.

An alternative route to produce graphene and chemically 45 modified graphene (CMG) is by the exfoliation of graphite or its derivatives, mainly graphite oxide (GO). The advantage of this approach is that it enables high yield production and, hence, it is a cost-effective and scalable process. ${ }^{9}$ These materials are, therefore, suitable for the production of polymer 50 nanocomposites.

Several methods have been reported for the exfoliation of graphite into graphene and CMG. They can be grouped according to the starting material in two groups: i) from graphite and ii) from GO; being the latter the most promising
55 and widely used in the literature. The reason for this preferential use of GO is related to the lower degree of exfoliation achieved by the graphite and expandable graphite, as evidenced by the presence of the graphite diffraction peak in the reported XRD patterns. ${ }^{10-12}$ The exfoliation of GO to ${ }_{60}$ graphene oxide can be readily done by a mild sonication in water but the resulting material is electrically insulating so it should be reduced to restore its conductivity. This reduction of GO to graphene or CMG have been carried out by either a chemical route or thermal treatment (Fig. 1, f and g). 65
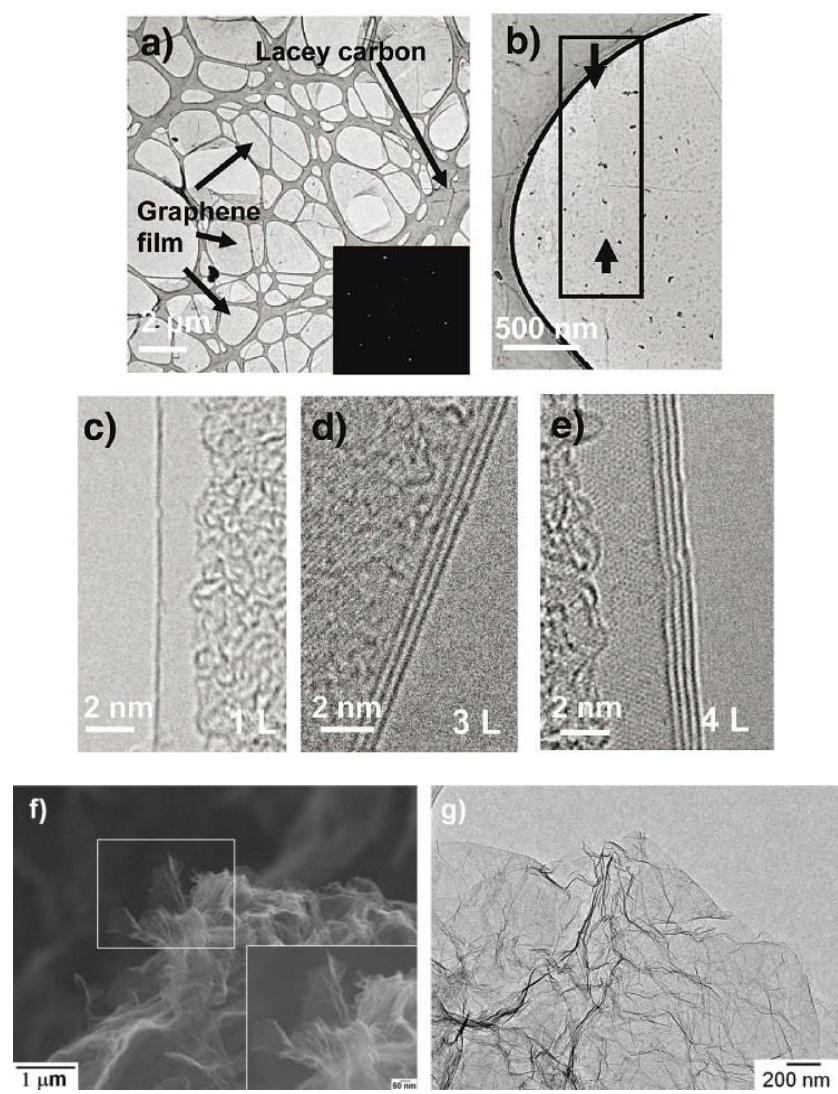

Fig. 1 Images of CVD and thermally exfoliated graphenes. (a-e) Low and high magnification TEM images of a CVD-grown graphene film (c-e)

70 high magnification TEM images showing the edges of film regions consisting of one, three, and four layers. Reprinted with permission from ${ }^{8}$ Copyright 2009 American Chemical Society. (f and g) SEM and TEM images of thermally exfoliated graphene showing its characteristic wrinkled structure. 
The focus of this review is on graphene filled polymer nanocomposites. Several recent reviews have already addressed graphene and chemically modified graphene synthesis, chemistry and properties ${ }^{3-5,9,13}$ and, hence, will not 5 be discussed in detail in this paper. We first review the current progress on the production procedures of the graphene/polymer nanocomposites. Then, the resultant properties and potential applications are also discussed. We will concentrate on both reduced and unreduced GO since the 10 vast majority of the studies on this subject used these two nanofillers. Some examples of CVD-grown graphenes have also been reported and will be briefly addressed. Finally, we will discuss the challenges and outlook of these emerging polymer composites.

\section{${ }_{15}$ 2. Production of Graphene/Polymer Nanocomposites}

A crucial step in polymer nanocomposites is the dispersion of the nanofillers. A well dispersed state ensures a maximised reinforced surface area, which will affect the neighbouring ${ }_{20}$ polymer chains and, consequently, the properties of the whole matrix. Therefore, large efforts have been concentrated on achieving a homogeneous and well-dispersed system by developing either covalent or non-covalent functionalisation of the filler surface. The reader interested in this subject is ${ }_{25}$ referred to reviews on CNT chemistry ${ }^{14}$ and graphene chemistry. ${ }^{9}, 13$

As discussed above, most graphene/polymer composites have been developed using both reduced and unreduced GO using three strategies: 1) solvent processing, 2) in situ 30 polymerisation and 3) melt processing.

\subsection{Solvent processing}

As already mentioned, GO is exfoliated into individual sheets by either a chemical route or a thermal treatment. The first procedure to exfoliate GO into graphene oxide layers takes 35 advantage of the presence of polar oxygen functional groups which enable the direct dispersion of GO in water and many organic solvents. These graphene oxide sheets are then reduced to recover the $\mathrm{sp}^{2}$ carbon network using solvents, such as hydrazine, dimethylhydrazine, sodium borohydride 40 and ascorbic acid. The reduction of the GO layers is partially achieved and the graphene sheets present acid groups on their surface. ${ }^{9}, 13$ The second procedure, the thermal expansion, enables the exfoliation and reduction of GO into graphene layers by a simple and rapid heat treatment. It also renders 45 partially oxygenated graphene layers that disperse readily in polar solvents. ${ }^{15}$ Hence, it is natural to use these dispersions to produce $\mathrm{CMG} /$ polymer composites via the solvent-blending procedure. The method consists in three steps: dispersion in a suitable solvent by, for example, ultrasonication, addition of 50 the polymer, and removal of the solvent by evaporation or distillation. Several composites have been described using this strategy both in aqueous media, such as nafion, ${ }^{16}$ or polystyrene (PS), ${ }^{17}$ and in organic solvents, such as PS, ${ }^{18}$ polyurethane (PU), ${ }^{19}$ poly(methyl methacrylate) (PMMA) ${ }^{15,} 20$ 55 or poly(styrene- $b$-isoprene- $b$-styrene) (SIS) block copolymer. $^{21}$
Due to the simplicity of this procedure, it is expected that graphene/polymer composites will continue to be developed using this methodology. However, a word of caution is in 60 place here as it has been reported that common organic solvents are strongly adsorbed on the graphitic galleries of GO in a permanent way. $^{22}$ Barroso-Bujans et al. systematically analysed both polar and non-polar solvents using solid state ${ }^{13} \mathrm{C}$ NMR and elemental analysis. They found 65 that all tried solvents penetrated and modified the graphitic layers of GO and, even after careful removal and drying protocol, traces of the solvents remained adsorbed on the materials. These observations make apparent the challenges and limitations of graphene chemistry in solvent-blending 70 procedures.

\subsection{In situ polymerisation}

In this strategy, the CMGs are mixed with the monomers or pre-polymers, sometimes in the presence of a solvent, and then the polymerisation reaction proceeds by adjusting 75 parameters such as temperature and time. Contrary to CNTs that require post-treatment, CMGs already present small molecules that could be used towards covalent bonding or towards further functionalisation such as grafting polymer chains via atom transfer radical polymerisation (ATRP). ${ }^{23}, 24$

${ }_{80}$ Examples of in situ polymerisation include $\mathrm{PU},{ }^{19} \mathrm{PS},{ }^{23-25}$ PMMA, ${ }^{24}$ epoxy, ${ }^{26,} 27$ or polydimethylsiloxane (PDMS) foams. ${ }^{28,29}$

Research on in situ polymerised nanocomposites should not only analyse the effect of the nanofillers in the polymer 85 matrix morphology and final properties but also in the polymerisation reaction or curing reaction. A study on this subject has revealed a decrease in the rate of the polymerisation reaction of PDMS foams by the addition of thermally exfoliated graphene and a change in the dynamic ${ }_{90}$ evolution of the reaction as compared to the effect of CNT. ${ }^{29}$ Changes in the molecular weight of TPU ${ }^{19}$ due to the presence of graphene have also been reported.

The advantages of this strategy are twofold: first, it provides a strong interaction between the incorporated ${ }_{95}$ particles and the polymer matrix, facilitating stress transfer, and second, it enables an outstanding and homogeneous dispersion. However, it is usually accompanied by a viscosity increase that hinders manipulation and loading fraction.

\subsection{Melt processing}

100 Melt processing is much more commercially attractive than the other two methods, as both solvent processing and in-situ polymerisation are less versatile and environmentally friendly. This strategy involves the direct inclusion of the CMGs into the melted polymer using a twin-screw extruder and adjusting 105 parameters such as screw speed, temperature and time. Most of the reported examples have been developed by mixing thermally exfoliated graphene with the following polymers: $\mathrm{PU},{ }^{19}$ isotactic polypropylene (iPP), ${ }^{30}$ poly(styrene-coacrylonitrile) $(\mathrm{SAN}),{ }^{30}$ polyamide $6 \quad(\mathrm{PA} 6)^{30}$ and 110 polycarbonate $(\mathrm{PC})^{30,} 31$ The drawbacks of this procedure are the low bulk density of thermally exfoliated graphene that makes extruder feeding a troublesome task and the lower 
degree of dispersion (Fig. 2) compared to solvent blending. ${ }^{19}$ This reduced dispersion degree will then result in poorer mechanical and transport properties as discussed in the following section.

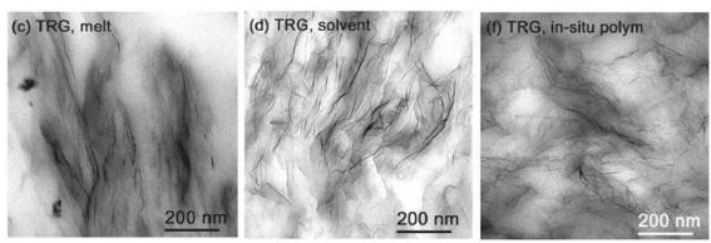

Fig. 2 Comparative images of the dispersion state using the three processing protocols. From left to right: TEM images of $3 \mathrm{wt} .-\%$ thermally exfoliated graphene in TPU by melt and solvent processing, and in situ polymerisation. The images clearly reveal the dispersion state of

10 the samples: melt processing samples present highly orientated thick

stacks, while both solvent and in situ polymerisation samples show homogeneously distributed thin sheets. Reprinted with permission from ${ }^{19}$ Copyright 2010 American Chemical Society.

\section{Properties}

${ }_{15}$ Due to the recent development of graphene and graphenepolymer nanocomposites, the literature on this subject is still in its early stages but developing rapidly. Nonetheless, several interesting studies have already been reported illustrating the potential of these new nanocomposites.

\section{${ }_{20}$ 3.1 Mechanical properties}

Most of the work on CNT/polymer nanocomposites is aimed at exploiting the remarkable mechanical properties of the nanotubes coupled with the possibility to introduce further functionalities, such as electrical conductivity or thermal 25 stability. $^{32}$ However, and despite all the research on $\mathrm{CNT}$ /polymer nanocomposites, several problems need to be resolved and the full potential has still to be reached.

The possibility of creating both structural and functional systems is even more feasible for graphene filled 30 nanocomposites due to the larger specific area, improved interfacial adhesion, and outstanding properties. ${ }^{18}$ The mechanical properties of perfect graphene sheets were recently measured by nanoindentation using an AFM, obtaining a Young's modulus of $1.0 \mathrm{TPa}$ and a fracture 35 strength of $130 \mathrm{GPa} .{ }^{33}$ Meanwhile, an elastic modulus of 0.25 $\mathrm{TPa}$ was obtained for chemically reduced graphene monolayers through tip-induced deformation experiments. ${ }^{34}$ As a comparison, Fig. 3 compiles the Young's modulus and densities of several materials.

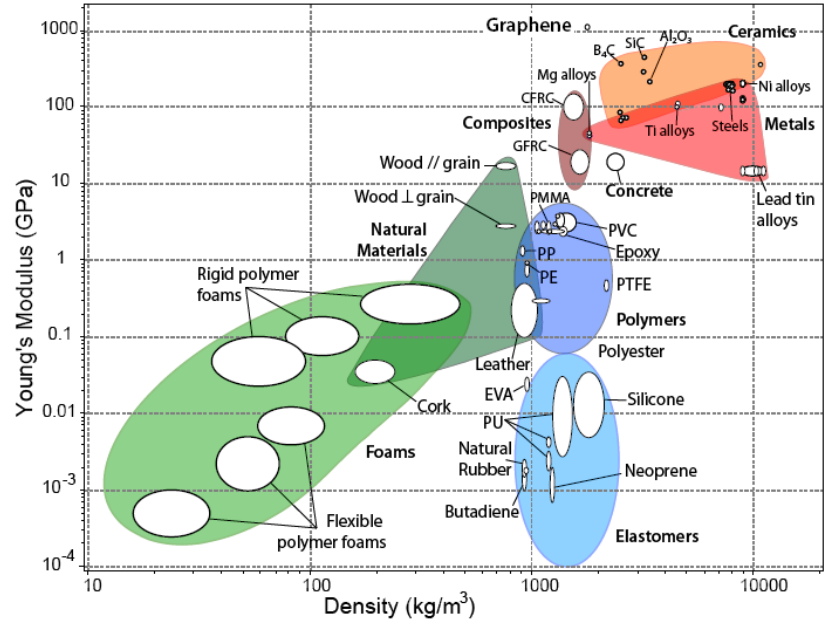

Fig. 3 Chart of Young's modulus as a function of density comparing graphene properties to more traditional materials. Note the axes are in logarithmic scale. Graphene density was taken as $2200 \mathrm{~kg} / \mathrm{m}^{3}$.

The studies focussing on the mechanical properties of 45 graphene filled polymer nanocomposites revealed an increase in modulus as a function of loading fraction. $15,19,20,25,27,28,30$, $31,35-40$ The modulus increase varied from up to $31 \%$ for 0.1 wt.- $\%$ thermally exfoliated graphene/epoxy, ${ }^{27} 120 \%$ for 1 wt.$\%$ chemically reduced graphene/TPU ${ }^{38}$ and $200 \%$ for 0.25 wt.$50 \%$ for thermally exfoliated graphene/silicone foam. ${ }^{28}$ As expected, the larger improvements were observed in elastomeric matrices due to their lower intrinsic modulus. Although the vast majority of the studies gave no details on the strength of the nanocomposites, some $27,37,38$ reported 55 increases of up to $75 \%$ with 0.7 wt. $-\%$ unreduced GO in PVA. $^{37}$ Additionally, thermally expanded graphene ${ }^{40}$ and graphite nanoplatelets ${ }^{41}$ remarkably reduced crack propagation in epoxy polymers. 

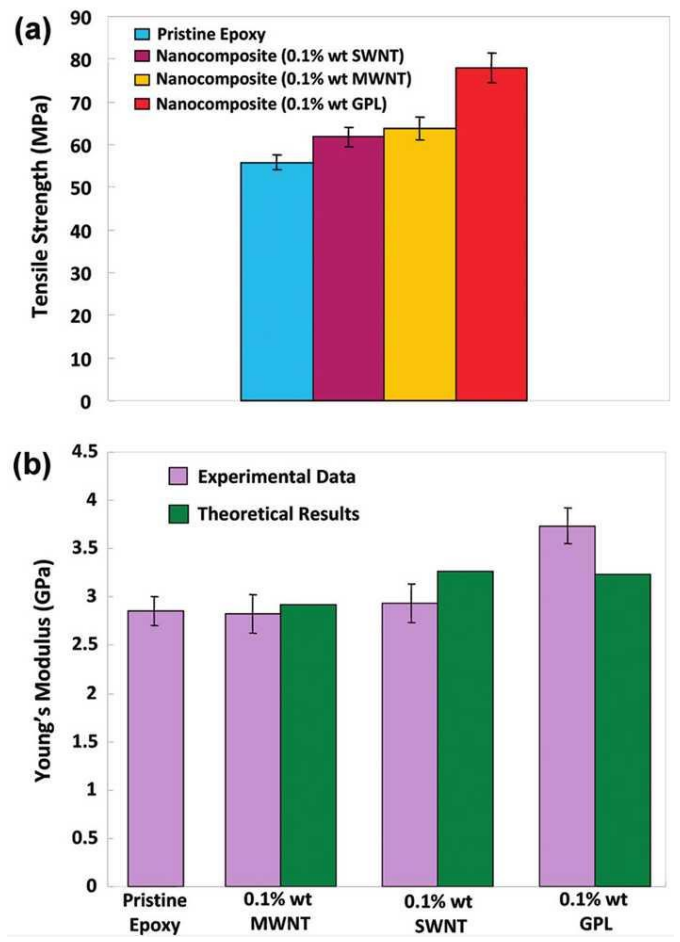

Fig. 4 Comparative study of the mechanical properties (uniaxial tensile tests) of an epoxy matrix filled with SWCNT, MWCNT and graphene. (a)

Tensile strength and (b) measured (left) and predicted (right) Young's 5 modulus of the unfilled epoxy and epoxy nanocomposites. The theoretical results were obtaining using the Halpin-Tsai theory. Reprinted with permission from. ${ }^{27}$ Copyright 2009 American Chemical Society.

Comparative studies of both CMG and CNT filled 10 nanocomposites carried out by Rafiee et al. ${ }^{27,} 40$ and Steurer et al. ${ }^{30}$ revealed greater improvements for CMG/polymer than for CNT/polymer systems (Fig. 4). The authors ascribed the results to the larger interfacial area and aspect ratio of CMG. We should point out that these two studies were done with 15 thermally exfoliated graphene which suggested that the presence of defects and wrinkles does not represent a disadvantage for mechanical reinforcement. Furthermore, a comparative study by Kim et al. ${ }^{19}$ on thermally and chemically exfoliated graphenes presented similar tensile 20 modulus increases. Also, Ramanathan et al. ${ }^{15}$ suggested that the presence of the wrinkles may actually lead to nanoscale surface roughness which would likely produce an enhanced mechanical interlocking and adhesion with the polymer chains (Fig. 5).
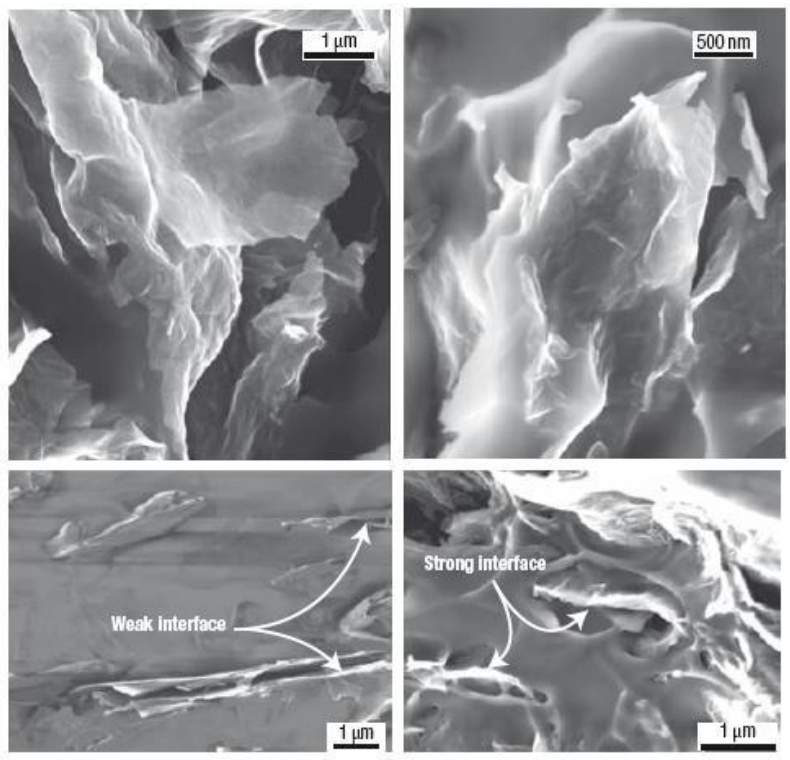

Fig. 5 Top: SEM images of a thermally exfoliated graphene/PMMA
acture surface revealing the surface roughness of the graphene. Bottom Comparative SEM images of expanded graphite/PMMA (left) and

thermally exfoliated graphene/PMMA (right) fracture surface. Reprinted 30 by permission from Macmillan Publishers Ltd: Nature Nanotechnology, ${ }^{15}$ copyright 2008

\subsection{Transport properties}

\subsubsection{Electrical conductivity}

Traditionally, insulating polymer matrices have been filled 35 with conductive fillers to develop lightweight electrically conductive materials or at least with resistivity lower than $10^{5}$ $\Omega /$ square corresponding to the anti-static range. These filled composite materials exhibit a non-linear increase of the electrical conductivity as a function of the filler concentration. 40 At certain loading fraction, known as percolation threshold, the fillers are able to form a network leading to a sudden rise of the electrical conductivity of the composite. ${ }^{42}$ The intrinsic high conductivity and aspect ratio of carbon-based nanofillers make them ideal candidates to achieve this percolated network 45 at low loading fractions.

Perfect graphene sheets have exhibited signs of ballistic transport. ${ }^{43}$ Though the electrical conductivity of CMGs is not as high as that of perfect graphene, it is still appropriate to develop electrically conductive nanocomposites. The first and 50 most widely used method for the chemical reduction and exfoliation of $\mathrm{GO}$ is through a colloidal dispersion in hydrazine monohydrate. ${ }^{13}$, 18 The obtained CMG retains carbonyl, epoxy, and carboxylic acid groups and the bulk conductivity of a powder sample was measured to be $2400 \pm$ ${ }_{55} 200 \mathrm{~S} / \mathrm{m}$, compared with $2500 \pm 20 \mathrm{~S} / \mathrm{m}$ for graphite and 0.021 $\pm 0.002 \mathrm{~S} / \mathrm{m}$ for $\mathrm{GO}$. The thermal procedure to reduce and exfoliate $\mathrm{GO}$ is through rapid heating at high temperatures. ${ }^{13}$, 44 The obtained CMG also retains carbonyl, epoxy, and carboxylic acid groups and presents a characteristic wrinkled ${ }_{60}$ damage structure. In spite of this defective surface, the bulk conductivities were measured to range from 1000-2300 S/m.

Although CNT have been shown to form an electrical percolated network at low loading fractions, initial studies have observed that graphene and $\mathrm{CMG}$ percolated at a 
similar $^{42}$ or even lower loading fraction. ${ }^{30}$

To illustrate this, we have collected the electrical percolation thresholds described in the literature and assembled the information in Fig. 6 according to the ${ }_{5}$ production methods: solvent processing, ${ }^{17}, 18,35,45-49$ in situ polymerisation ${ }^{19,36,50-53}$ and melt processing. ${ }^{19,30,31,54}$ As it can be seen, the percolation thresholds vary over a wide range of loading fractions, from the lowest at 0.07 vol.-\% for ultrahigh molecular weight $\mathrm{PE}^{47}$ and 0.1 vol.- $\%$ for $\mathrm{PS}^{18}$ to 3.8 10 vol.- $\%$ for polyamide $6 .{ }^{30}$ The differences arise from the type and functionalisation of graphene or CMG and from the degree of dispersion.

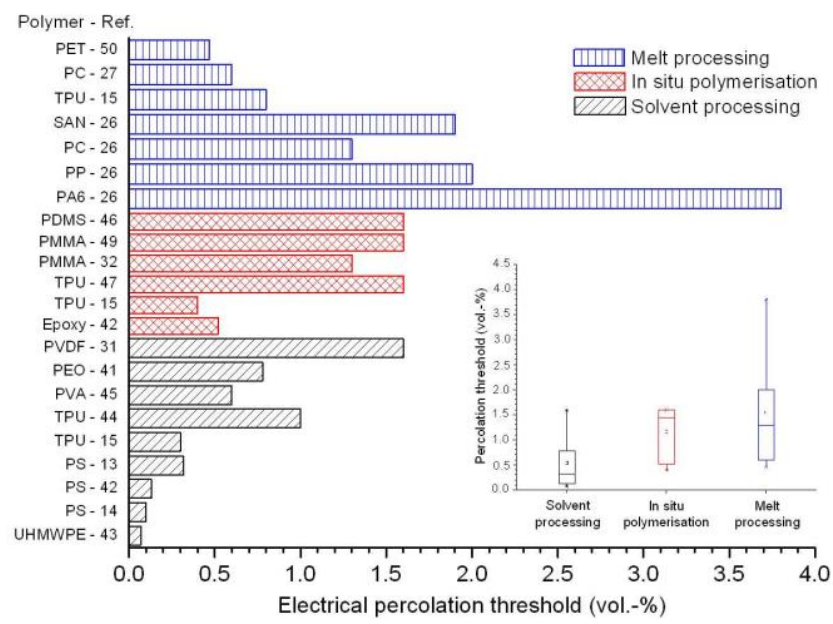

Fig. 6 Electrical percolation thresholds of graphene/polymer 15 nanocomposites according to processing strategy. For comparison purposes, the values of loading fraction reported in wt.- $\%$ have been converted to vol.-\%.

Low percolation thresholds were achieved with chemically reduced GO, using hydrazine ${ }^{47}$ and isocyanate ${ }^{18}$ via solvent 20 processing, while the largest was attained with a thermally exfoliated $\mathrm{GO}^{30}$ via melt processing. On average (Fig. 6, inset), the lowest percolation threshold was obtained by solvent processing, closely followed by in situ polymerisation since these two methods usually enabled better dispersions 25 than melt compounding. ${ }^{19}$ Also, the use of highly defective thermally exfoliated CMG could account for the higher percolation threshold. However, percolation threshold as low as 0.3 vol. $-\%$ in TPU was reported with thermally exfoliated graphene via solvent processing. ${ }^{19}$ In our opinion, the 30 differences among solvent and in situ polymerisation could arise from the intimate contact between the graphene sheet and the polymer chains attained by in situ polymerisation which effectively coats the sheet and shields it from the others.

35

\subsubsection{Thermal conductivity}

As opposed to the above discussed electrical conductivity, the thermal conductivity of graphene/polymer nanocomposites has received minimal attention. The reason may be related to 40 the modest improvements observed in CNT/polymer nanocomposites as compared to electrical conductivity enhancements of several orders of magnitude. ${ }^{42}$ The differences are related to the transport nature of electrical and thermal conduction. While electrical charge flows and can 45 tunnel through barriers, thermal energy is transmitted by the interaction of adjacent particles through a combination of vibrations and free electrons. Hence, good thermal conductivity requires a strong filler/polymer interface. Therefore, it is not surprising that in the studies describing a 50 positive effect of $\mathrm{CMG}$ in the thermal conductivity the nanocomposites were produced by in situ polymerisation. ${ }^{25,} 28$ Fang et al. ${ }^{25}$ reported an increase in the thermal conductivity of PS films filled with 2 wt.- $\%$ polystyrene-grafted graphene from $0.158 \mathrm{Wm}^{-1} \mathrm{~K}^{-1}$ to $0.413 \mathrm{Wm}^{-1} \mathrm{~K}^{-1}$. Silicone foams filled 55 with thermally exfoliated graphene produced by in situ polymerisation, reactive foaming displayed a $6 \%$ increase of the thermal conductivity at a loading fraction of $0.25 \mathrm{wt} .-\%$, due to the covalent bonding between the hydroxyl groups of the graphene and $\mathrm{SiH}$ - groups of the prepolymer. ${ }^{28}$ However, 60 part of this increase was also attributable to the indirect effect of the graphenes on the porous microstructure and foam density.

\subsection{Thermal properties and morphology}

${ }_{65}$ Several studies have evaluated the effect of $\mathrm{CMG}$ on the thermal properties, such as thermal stability, glass transition $\left(\mathrm{T}_{\mathrm{g}}\right)$ and melting $\left(\mathrm{T}_{\mathrm{m}}\right)$ temperatures, and polymer crystallinity of the nanocomposites.

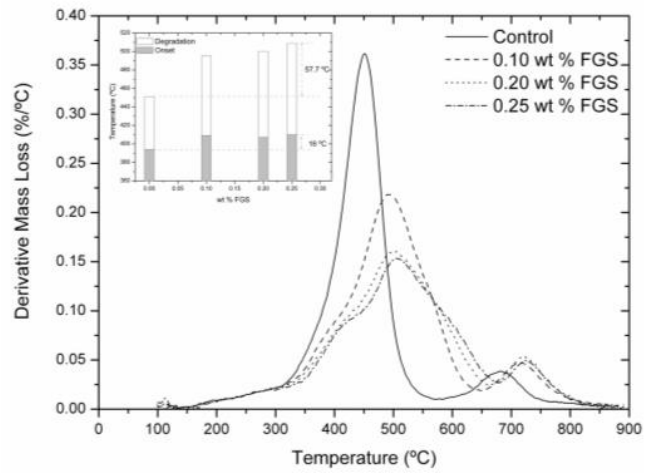

70 Fig. 7 Representative thermogravimetric results showing all three aspects of the degradation behaviour of CMG/silicone foams: (1) a $16{ }^{\circ} \mathrm{C}$ increase of the onset temperature (inset), (2) a nearly $60^{\circ} \mathrm{C}$ increase of the degradation temperature (inset), and (3) reduced degradation rate. ${ }^{28}$

Polymers degrade at low temperatures which limits their 75 uses in high temperature applications as compared to ceramics or metals. The degradation behaviour of polymers is commonly evaluated in terms of three parameters: (1) the onset temperature, considered as the temperature at which the system starts to degrade, (2) the degradation temperature, 80 considered as the temperature at which the maximum degradation rate occurs, and (3) the degradation rate, seen in the derivative weight loss as a function of temperature curve. Two opposite trends have experimentally been observed for the thermal stability behaviour of CMG nanocomposites. ${ }_{85}$ While both thermally ${ }^{15}, 28,55$ and chemically ${ }^{49}$ reduced GO improved the thermal decomposition temperature (Fig. 7) of several polymer matrices, non-reduced GO did not significantly affected the thermal stability of several polymer 
matrices. ${ }^{56}$ The improved thermal stability of the CMG/polymer nanocomposites was attributed to the high surface area and good dispersion of the nanosheet filler and strong graphene/polymer interactions. ${ }^{15,28,49,55}$

5 Non-crystalline materials, and in particular polymers, exhibit a second order phase transition, called the glass transition temperature $\left(\mathrm{T}_{\mathrm{g}}\right)$, where the material changes from a brittle, crystalline (or semi-crystalline) solid to an elastic, amorphous solid. Several studies have reported a drastic 10 increase of the glass transition temperature in a thermally exfoliated GO/PMMA $\left(30{ }^{\circ} \mathrm{C}\right.$ at 0.05 wt.- $\left.\%\right)$, ${ }^{15}$ a hydrazine reduced GO/PVA $\left(20{ }^{\circ} \mathrm{C} \text { at } 10 \text { wt.- } \%\right)^{49}$ and a PS-grafted GO/PS $\left(15{ }^{\circ} \mathrm{C}\right.$ at 12 wt.- $\left.\%\right) .{ }^{25}$ These differences could be associated to the suggested improvement mechanisms. PVA 15 and PS chains were considered to present a reduced mobility via covalent bonds to the CMG sheets. Meanwhile, PMMA reduced mobility was attributed to two mechanisms: a) mechanical interlocking and adhesion due to the wrinkled topology of the thermally exfoliated GO, and b) hydrogen 20 bonding between the polymer chains and the oxygen functionalities of the CMG sheets.

Contradictory results have also been reported on the crystallinity of graphene/polymer nanocomposite. While the degree of crystallisation was observed to increase in 25 exfoliated graphene/PVA, ${ }^{57}$ no effect was observed in $\mathrm{GO} / \mathrm{PVA}^{37}$ and the complete opposite effect was observed in chemically reduced GO/PVA. ${ }^{49}$ In our opinion, the likely explanation lies on the nature of polymer crystallisation as it is heavily influenced by the thermal history, the 30 manufacturing process employed, and the interfacial interaction of the fillers.

\section{Potential Applications}

The envisioned applications of graphene filled polymer composites rely on the above discussed properties. ${ }_{35}$ Graphene/polymer composites are currently starting to appear and, thus, the potential applications of these new materials are still open for further research. In this context, research on this area will benefit from the large body of research done on $\mathrm{CNT} /$ polymer nanocomposites and we expect a rapid 40 development on this subject.

The reader should be aware that although some of the applications discussed below have already been proposed and studied on graphene filled polymer nanocomposites, others come from the CNT/polymer nanocomposite field and are yet 45 to be studied. Several CVD grown graphene/polymer nanocomposite applications will be discussed throughout this section. We decided to separate the potential applications according to structural and functional reinforcement, depending on whether the intended improvement was to 50 strengthen the initial material, or to provide an added-value property. However, we should point out that this distinction is becoming blurred in polymer nanocomposites and several examples of the structural section could well be regarded as functional reinforcement.

\subsection{Structural reinforcement}

The improvement on the mechanical properties of polymer matrices suggested the use of these materials in transport applications, where the combination of high strength and light 60 weight provide environmental advantages for enhanced fuelefficiency vehicles. ${ }^{58}$

Additionally, the use of nanofillers opened up the possibility to reinforce delicate systems where conventional fibres cannot be physically accommodated, such as thin films, 65 fibres and foams:

i) Thin films find applications as membranes in many areas from the petrochemical to biomedical industries. The platelet morphology of graphene and CMG and the fact that perfect graphene sheet from micromechanical cleavage has been observed to be impermeable even to $\mathrm{He}$ gas $^{59}$ could make possible membranes with enhanced permittivity and selectivity. Initial studies on TPU films have shown lower permeability values of both thermally and chemically exfoliated graphene than organically modified montmorillonite layers at similar loadings. ${ }^{19}$

ii) Reinforced polymer fibres can be prepared from solution and melt compounding methods such as melt spinning method or electrospinning. Potential applications of fibre nanocomposites range from smart fabrics and woven materials $^{60}$ to optical fibres. ${ }^{61,} 62$ A fibre made by a diphenyl sulfide assisted dispersion of CNT in PMMA was applied as a saturable absorber for passively modelocked lasing device. ${ }^{61}$ Recently, a membrane made of electrospun CMG/poly(vinyl acetate) fibres also demonstrated its capability as a saturable absorber with wideband absorption and as efficient photonic material for the generation of ultra-short pulses in fibre lasers. ${ }^{62}$

iii) Polymer foams are versatile materials used widely in transport, construction, packaging or biomedical applications. The incorporation of CNT to polymer foams have shown to improve simultaneously several properties, such as the mechanical performance, surface characteristics, electromagnetic interference (EMI) shielding characteristics, acoustic insulation and flammability. ${ }^{63-67}$ When used in biomedical applications, CNT filled polymer foams or scaffolds modified the cell phenotype, which is relevant for medical regeneration therapies. ${ }^{63}, 65$ Initial studies of thermally exfoliated graphene in polymer foams demonstrated the simultaneous enhancement of the compression strength, with an increase in the normalised modulus of more than $200 \%$, and thermal stability, with an increase of the decomposition temperature of nearly $60{ }^{\circ} \mathrm{C}$, with loading fractions as low as 0.25 wt. $-\%{ }^{28} \mathrm{~A}$ comparative analysis between thermally exfoliated GO and CNT revealed that the CMG offered greater improvement on the thermal stability of the foams attributed to an increased confinement of the polymer chains in the intercalated structure and to improved graphene/polymer interactions. ${ }^{55}$ This result could further suggest the potential applicability of CMG as flame-retardant filler since CNT, with a similar thermal stability improvement, created a protective network along the cellular structure resulting on a self-extinguishing grade system. ${ }^{64}$ 


\subsection{Functional reinforcement}

The intrinsic high conductivity and aspect ratio of graphene enable reaching the percolation threshold at lower loading fractions than carbon black and multiwall carbon nanotubes. ${ }^{30}$

5 The achieved conductivity determines the applications of the conducting polymers, from sensors to electrostatic discharge (ESD) and EMI shielding materials, and electrodes.

\subsubsection{Sensors}

${ }_{10}$ Sensors could be made out of materials with a wide range of conductivities, from polymer composites close to the percolation threshold ${ }^{68}$ to electrically conductive polymers, ${ }^{69}$ finding applications as gas, ${ }^{69} \mathrm{pH},{ }^{70}$ pressure ${ }^{68}$ or temperature ${ }^{35}$ sensors.

15 Conductive polymer nanocomposites usually exhibit a positive temperature coefficient, i.e. the resistivity increases with temperature. However, a recent study described the opposite behaviour (negative temperature coefficient) in PVDF nanocomposites filled with thermally reduced ${ }_{20}$ graphene. ${ }^{35}$ This unexpected result was attributed to contact resistance predominating over tunneling resistance due to the large aspect ratio of these graphene sheets compared to graphite nanoparticles. Such negative temperature coefficient is common in commercially available temperature sensors 25 based in ceramic thermistors and, hence, could potentially serve as an alternative technology.

\subsubsection{ESD and EMI shielding materials}

ESD materials are characterised by a surface resistivity 30 between $10^{12}$ and $10^{5} \Omega$ /square, while EMI shielding materials should have a resistivity lower than $10^{5} \Omega /$ square. The applications of ESD and EMI materials go from carpeting floor mats, and electronics packaging to telecommunication antenna, mobile phone parts and frequency shielding coatings 35 for aircraft and electronics. EMI shielding efficiency has already been measured in an CMG/epoxy nanocomposite, achieving a commercially suitable level (around $20 \mathrm{~dB}$ in the $\mathrm{X}$-band) for 15 wt.-\% loading fraction of hydrazine reduced GO. ${ }^{52}$

40

\subsubsection{Electrodes}

The most widely studied applications of graphene and CMG is as electrodes, in lithium-ion batteries, ${ }^{71}$ supercapacitors, ${ }^{72-74}$ organic solar cells, ${ }^{75-78}$ and field emission devices: ${ }^{79,} 80$

${ }_{45}$ i) Activated carbon is currently used as electrodes in most commercially available supercapacitors, or electrochemical double-layer capacitor due to high surface area and electronic conductivity. ${ }^{81}$ One approach to improve the performances of these electrodes was to combine carbon-based nanofillers with pseudocapacitive materials, such as polymers. On-going research on this area has reported specific capacitances ranging from 120 $\mathrm{F} / \mathrm{g}$ (scan rate $5 \mathrm{mV} / \mathrm{s}$ ) for a thermally reduced graphene /propylene carbonate ${ }^{74}$ using tetraethylammonium

55 tetrafluoroborate as electrolyte, to $408 \mathrm{~F} / \mathrm{g}$ (scan rate 5 $\mathrm{mV} / \mathrm{s}$ ) for a nanocomposite of polyaniline (PANI) filled with a reduced $\mathrm{GO}$ by microwave-assisted solvothermal method $^{72}$ using $\mathrm{H}_{2} \mathrm{SO}_{4}$ as electrolyte. Recently, the combination of hydrazine reduced GO with CNT in PANI exhibited a specific capacitance of $1035 \mathrm{~F} / \mathrm{g}$ (scan rate 1 $\mathrm{mV} / \mathrm{s}$ ) in $\mathrm{KOH}$ as electrolyte. Additionally, this hybrid CNT/CMG system offered long-term stability, measured during 1000 charge/discharge cycles, with a capacitance decreased of merely $6 \%$ of its initial value. ${ }^{73}$

${ }_{65}$ ii) Transparent conductive films are used in many electronic devices, such as solar cells, touch screen, flat panel displays, etc. Both CVD grown graphene and CMG have been used for the development of such films following different approaches. CVD graphene are synthesised on metal substrates and are then transferred to different transparent films (Fig. 8), such as PDMS, ${ }^{82}$ or PET. ${ }^{75,80,83}$ This approach has provided lower film sheet resistances for similar transparency values than CMG and has outperformed current commercially available technologies. ${ }^{75}$ These films have been subsequently tested for solar cells applications, ${ }^{75}$ field-effect transistor, ${ }^{82}$ and touch-screen panels. $^{83}$ On the other hand, CMG transparent films can be easily prepared by several methods, such as spin-casting, ${ }^{77,} 84$ dip-coating ${ }^{78}$ and Langmuir-Blodgett assembly. ${ }^{85}$ Although the sheet resistance of the CMG films was considerably higher than the CVD method due to the presence of surface functionalities and defects, its scalability was considered to be more straightforward. However, a recent development by Hong and co-workers used a roll-to-roll production, similar to a newspaper printing press, to transfer CVD grown graphene onto different substrates opening up the scale up process of CVD graphene films. ${ }^{83}$

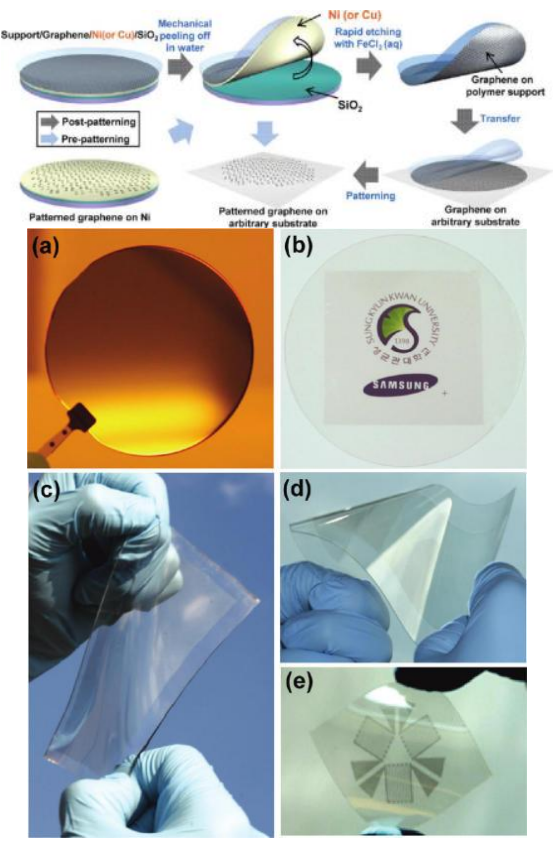

Fig. 8 Top: Schematic illustration of the synthesis, etching, and transfer of large-area graphene films. Bottom: Photographs of (a) as-grown and transferred wafer-scale graphene films $(b, d)$ on a PET substrate and (c) on a stretchable rubber substrate. (e) A strain gauge pattern on rubber by 95 pre-patterning method. Reprinted with permission from. ${ }^{82}$ Copyright 2010 American Chemical Society. 


\subsubsection{Stimuli responsive materials}

A further step to multifunctional polymer nanocomposites is the development of systems that respond to external stimuli, such as temperature, electric field, light, and $\mathrm{pH}$, among 5 others. Responsive graphene/polymer nanocomposites have already been described with three different external stimuli, light $^{38}$, electrical field, ${ }^{86}$ and temperature. ${ }^{41}$ TPU nanocomposites with $1 \mathrm{wt} .-\%$ sulfonated-graphene exhibited a repeatable infrared-triggered actuation performance. The 10 nanocomposites could contract and lift a $21.6 \mathrm{~g}$ weight $3.1 \mathrm{~cm}$ with $0.21 \mathrm{~N}$ of force on exposure to infrared light. ${ }^{38} \mathrm{Xiao}$ et al. ${ }^{41}$ did not use single layer graphene but rather CVD grown graphite nanoparticles made of stacks of less than 10 layers. They then analysed the shape memory of an epoxy resin by 15 performing scratch tests at room temperature and then heating up the sample above its $\mathrm{T}_{\mathrm{g}}$ (Fig. 9). The scratches on the filled samples almost completely disappeared after heating, which was ascribed to the resilience to crack formation of the filled samples.

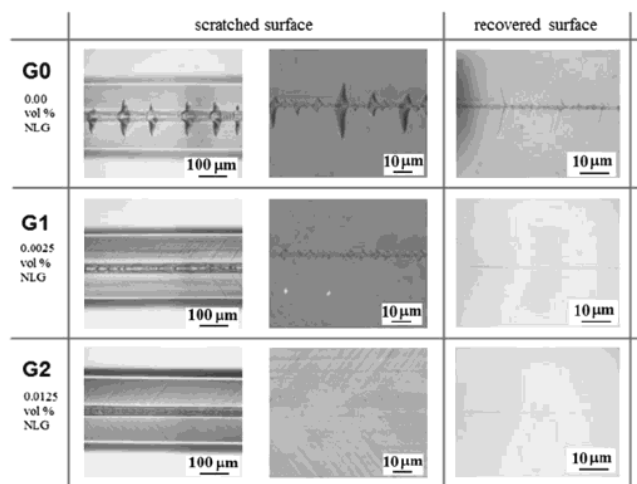

Fig. 9 Optical images of the as-scratched and recovered polymer surfaces of unfilled (top row) and filled samples. ${ }^{41}$ The images illustrate the permanent damage observed in the unfilled sample and the almost completely disappeared in the filled samples. Reproduced by permission 25 of The Royal Society of Chemistry.

\section{Conclusions and Outlook}

We have reviewed the current progress on the production, properties and potential applications of graphene filled polymer nanocomposites. Although this field is still in an 30 early stage of development, it has become apparent its growing interest and huge potential. However, we should learn from the unfulfilled expectations of $\mathrm{CNT} /$ polymer nanocomposites and keep into perspective the challenges and fundamental issues needed to be resolved.

35 The first challenge relates to graphene and CMG production. The preparation and transfer of high quality graphene is still not viable in a cost effective manner, although we have recently witnessed a promising step forward in this direction. ${ }^{83}$ While it is possible to scale up the current 40 production of CMG, it is necessary to develop further understanding and, hence control, on the process. This control would enable us to grasp physics of defect formation and, subsequently, maximise the restoration of the $\mathrm{sp}^{2}$ carbon network. Also, the development of new synthesis routes 45 should be explored to overcome the current risks of explosion and reduce acid and solvent wastes.
The second immediate challenge relates to the composite processing. The full exploitation of graphene filled nanocomposites will be determined both by the level of 50 graphene dispersion and alignment, and by the cost-effective manufacturing of the final material. Graphene dispersion and orientation is crucial to optimise its effectiveness for both structural and functional performance. Several studies have already demonstrated the potential of functionalisation to 5 develop strong interfacial bonding between graphene and $\mathrm{CMG}$ and the polymer matrix. However, we still lack an understanding of the effects of the graphene/polymer interactions in the matrix conformation, crystallinity and final properties. Further work in this area will improve the adhesion ${ }_{60}$ between graphene and polymers leading to enhanced stress transfer, reduced thermal resistance and reduced interfacial free volume. Additionally, going beyond random orientation of the nanofiller would lead to the development of "nanocomposites-by-design" with precise control of the 65 morphology. 87

\section{Acknowledgements}

The authors of this study gratefully acknowledge the financial support from the Spanish Ministry of Science and Innovation (MiCInn) under project MAT 2010-18749. RV and MMB also 70 acknowlonge the Ramon $y$ Cajal and FPI programme, respec $\overline{\overline{i v}}$ ly.

\section{Notes and References}

Institute of Polymer Science and Technology, (CSIC), C/ Juan de la Cierva, 3, 28006 Madrid, Spain Fax: +34 91 5644853; Tel: +34 91258 75 7424; E-mail: rverdejo@ictp.csic.es

1. R. A. Vaia and H. D. Wagner, Mater Today, 2004, 7, 32-37.

2. N. A. Kotov, Nature, 2006, 442, 254-255.

3. A. K. Geim and K. S. Novoselov, Nat Mater, 2007, 6, 183-191.

80 4. C. N. R. Rao, K. Biswas, K. S. Subrahmanyam and A. Govindaraj, J. Mater. Chem., 2009, 19, 2457-2469.

5. C. Soldano, A. Mahmood and E. Dujardin, Carbon, 2010, 48, $2127-$ 2150.

6. K. S. Novoselov, A. K. Geim, S. V. Morozov, D. Jiang, Y. Zhang, S.

85 V. Dubonos, I. V. Grigorieva and A. A. Firsov, Science, 2004, 306, 666-669.

7. A. Dato, V. Radmilovic, Z. Lee, J. Phillips and M. Frenklach, Nano Lett, 2008, 8, 2012-2016.

8. A. Reina, X. Jia, J. Ho, D. Nezich, H. Son, V. Bulovic, M. S.

90 Dresselhaus and J. Kong, Nano Lett, 2009, 9, 30-35.

9. S. Park and R. S. Ruoff, Nat. Nanotechnol., 2009, 4, 217-224.

10. G. Chen, D. Wu, W. Weng and C. Wu, Carbon, 2003, 41, 619-621.

11. T. Ramanathan, S. Stankovich, D. A. Dikin, H. Liu, H. Shen, S. T. Nguyen and L. C. Brinson, J. Polym. Sci., Part B: Polym. Phys., $95 \quad 2007,45,2097-2112$

12. F. M. Uhl, Q. Yao and C. A. Wilkie, Polym. Adv. Technol., 2005, 16, 533-540.

13. D. R. Dreyer, S. Park, C. W. Bielawski and R. S. Ruoff, Chem. Soc. Rev., 2010, 39, 228-240.

100 14. D. Tasis, N. Tagmatarchis, A. Bianco and M. Prato, Chem. Rev., 2006, 106, 1105-1136. 
15. T. Ramanathan, A. A. Abdala, S. Stankovich, D. A. Dikin, M. Herrera-Alonso, R. D. Piner, D. H. Adamson, H. C. Schniepp, X. Chen, R. S. Ruoff, S. T. Nguyen, I. A. Aksay, R. K. Prud'homme and L. C. Brinson, Nat. Nanotechnol., 2008, 3, 327-331.

5 16. S. Ansari, A. Kelarakis, L. Estevez and E. P. Giannelis, Small, 2010, 6, 205-209.

17. E. Tkalya, M. Ghislandi, A. Alekseev, C. Koning and J. Loos, J. Mater. Chem., 2010, 20, 3035-3039.

18. S. Stankovich, D. A. Dikin, G. H. B. Dommett, K. M. Kohlhaas, E. J. Zimney, E. A. Stach, R. D. Piner, S. T. Nguyen and R. S. Ruoff, Nature, 2006, 442, 282-286.

19. H. Kim, Y. Miura and C. W. Macosko, Chem. Mat., 2010, 22, 34413450.

20. B. Das, K. E. Prasad, U. Ramamurty and C. N. R. Rao,

15 Nanotechnology, 2009, 20, 125705/125701-125705/125705.

21. L. Peponi, A. Tercjak, R. Verdejo, M. A. Lopez-Manchado, I. Mondragon and J. M. Kenny, J. Phys. Chem. C, 2009, 113, 1797317978.

22. F. Barroso-Bujans, S. Cerveny, R. Verdejo, J. J. d. Val, J. M. Alberdi,

20 A. Alegria and J. Colmenero, Carbon, 2010, 48, 1079 - 1087.

23. M. Fang, K. G. Wang, H. B. Lu, Y. L. Yang and S. Nutt, J. Mater. Chem., 2010, 20, 1982-1992.

24. S. H. Lee, D. R. Dreyer, J. H. An, A. Velamakanni, R. D. Piner, S. Park, Y. W. Zhu, S. O. Kim, C. W. Bielawski and R. S. Ruoff,

25 Macromol. Rapid Commun., 2010, 31, 281-288.

25. M. Fang, K. G. Wang, H. B. Lu, Y. L. Yang and S. Nutt, J. Mater. Chem., 2009, 19, 7098-7105.

26. S. R. Wang, M. Tambraparni, J. J. Qiu, J. Tipton and D. Dean, Macromolecules, 2009, 42, 5251-5255.

30 27. M. A. Rafiee, J. Rafiee, Z. Wang, H. Song, Z.-Z. Yu and N. Koratkar, ACS Nano, 2009, 3, 3884-3890.

28. R. Verdejo, F. Barroso-Bujans, M. A. Rodriguez-Perez, J. A. de Saja and M. A. Lopez-Manchado, J. Mater. Chem., 2008, 18, 2221-2226.

29. R. Verdejo, F. J. Tapiador, L. Helfen, M. M. Bernal, N. Bitinis and

35 M. A. Lopez-Manchado, Phys. Chem. Chem. Phys., 2009, 11, 1086010866.

30. P. Steurer, R. Wissert, R. Thomann and R. Mulhaupt, Macromol. Rapid Commun., 2009, 30, 316-327.

31. H. Kim and C. W. Macosko, Polymer, 2009, 50, 3797-3809.

40 32. P. M. Ajayan and J. M. Tour, Nature, 2007, 447, 1066-1068.

33. C. Lee, X. D. Wei, J. W. Kysar and J. Hone, Science, 2008, 321, $385-$ 388.

34. C. Gómez-Navarro, M. Burghard and K. Kern, Nano Letters, 2008, 8, 2045-2049.

45 35. S. Ansari and E. P. Giannelis, J. Polym. Sci. Pt. B-Polym. Phys., 2009, 47, 888-897.

36. J. Y. Jang, M. S. Kim, H. M. Jeong and C. M. Shin, Compos. Sci. Technol., 2009, 69, 186-191.

37. J. J. Liang, Y. Huang, L. Zhang, Y. Wang, Y. F. Ma, T. Y. Guo and

$50 \quad$ Y. S. Chen, Adv. Funct. Mater., 2009, 19, 2297-2302.

38. J. J. Liang, Y. F. Xu, Y. Huang, L. Zhang, Y. Wang, Y. F. Ma, F. F. Li, T. Y. Guo and Y. S. Chen, J. Phys. Chem. C, 2009, 113, 99219927.

39. D. A. Nguyen, Y. R. Lee, A. V. Raghu, H. M. Jeong, C. M. Shin and

55 B. K. Kim, Polym. Int., 2009, 58, 412-417.
40. M. A. Rafiee, J. Rafiee, I. Srivastava, Z. Wang, H. Song, Z.-Z. Yu and N. Koratkar, Small, 2010, 6, 179-183.

41. X. Xiao, T. Xie and Y. T. Cheng, J. Mater. Chem., 2010, 20, 35083514.

60 42. M. Moniruzzaman and K. I. Winey, Macromolecules, 2006, 39, 5194-5205.

43. X. Du, I. Skachko, A. Barker and E. Y. Andrei, Nat Nanotechnol, 2008, 3, 491-495.

44. H. C. Schniepp, J. L. Li, M. J. McAllister, H. Sai, M. Herrera-

65 Alonso, D. H. Adamson, R. K. Prud'homme, R. Car, D. A. Saville and I. A. Aksay, Journal of Physical Chemistry B, 2006, 110, 85358539.

45. H. B. Lee, A. V. Raghu, K. S. Yoon and H. M. Jeong, J. Macromol. Sci. Part B-Phys., 2010, 49, 802-809.

70 46. N. Liu, F. Luo, H. Wu, Y. Liu, C. Zhang and J. Chen, Adv. Funct. Mater., 2008, 18, 1518-1525.

47. H. Pang, T. Chen, G. Zhang, B. Zeng and Z.-M. Li, Materials Letters, 2010, 64 2226-2229.

48. A. V. Raghu, Y. R. Lee, H. M. Jeong and C. M. Shin, 75 Macromolecular Chemistry and Physics, 2008, 209, 2487-2493.

49. H. J. Salavagione, G. Martinez and M. A. Gomez, J. Mater. Chem., 2009, 19, 5027-5032.

50. M. Kujawski, J. D. Pearse and E. Smela, Carbon, 2010, 48, 24092417.

80 51. Y. R. Lee, A. V. Raghu, H. M. Jeong and B. K. Kim, Macromolecular Chemistry and Physics, 2009, 210, 1247-1254.

52. J. J. Liang, Y. Wang, Y. Huang, Y. F. Ma, Z. F. Liu, F. M. Cai, C. D. Zhang, H. J. Gao and Y. S. Chen, Carbon, 2009, 47, 922-925.

53. W. Wen-Ping and P. Cai-Yuan, Polymer Engineering \& Science, 85 2004, 44, 2335-2339.

54. H. B. Zhang, W. G. Zheng, Q. Yan, Y. Yang, J. W. Wang, Z. H. Lu, G. Y. Ji and Z. Z. Yu, Polymer, 2010, 51, 1191-1196.

55. R. Verdejo, C. Saiz-Arroyo, J. Carretero-Gonzalez, F. BarrosoBujans, M. A. Rodriguez-Perez and M. A. Lopez-Manchado, Eur. $90 \quad$ Polym. J., 2008, 44, 2790-2797.

56. A. L. Higginbotham, J. R. Lomeda, A. B. Morgan and J. M. Tour, ACS Appl Mater Interfaces, 2009, 1, 2256-2261.

57. K. E. Prasad, B. Das, U. Maitra, U. Ramamurty and C. N. R. Rao, Proc. Natl. Acad. Sci., 2009, 106, 13186-13189.

95 58. E. V. Barrera, M. L. Shofner and E. L. Corral, in Carbon Nanotubes: Science and Applications ed. M. Meyyappan, CRC Press, Boca Raton, Florida, pp. 253-275.

59. J. S. Bunch, S. S. Verbridge, J. S. Alden, A. M. v. d. Zande, J. M. Parpia, H. G. Craighead and P. L. McEuen, Nano Lett, 2008, 8, 24582462.

60. G. G. Wallace, Fibers and Polymers, 2007, 8, 135-142.

61. S. Uchida, A. Martinez, Y. W. Song, T. Ishigure and S. Yamashita, Opt. Lett., 2009, 34, 3077-3079.

62. Q. Bao, H. Zhang, J.-x. Yang, S. Wang, D. Y. Tang, R. Jose, S.

105 Ramakrishna, C. T. Lim and K. P. Loh, Adv. Funct. Mater., 2010, 20, 782-791.

63. G. Jell, R. Verdejo, L. Safinia, M. Stevens, M. Shaffer and A. Bismarck, J. Mater. Chem., 2008, 18, 1865-1872.

64. R. Verdejo, F. Barroso-Bujans, M. A. Rodriguez-Perez, J. A. de Saja, 110 M. Arroyo and M. A. Lopez-Manchado, J. Mater. Chem., 2008, 18, 3933-3939. 
65. R. Verdejo, G. Jell, L. Safinia, A. Bismarck, M. M. Stevens and M. S. P. Shaffer, J. Biomed. Mater. Res. A, 2009, 88A, 65-73.

66. R. Verdejo, R. Stämpfli, M. Alvarez-Lainez, S. Mourad, M. A. Rodriguez-Perez, P. Brühwiler and M. Shaffer, Compos. Sci.

$5 \quad$ Technol., 2009, 69, 1564-1569.

67. Y. Yang, M. C. Gupta, K. L. Dudley and R. W. Lawrence, Nano Lett., 2005, 5, 2131-2134.

68. L. Chen, G. Chen and L. Lu, Adv. Funct. Mater., 2007, 17, 898-904.

69. K. H. An, S. Y. Jeong, H. R. Hwang and Y. H. Lee, Adv. Mater., $10 \quad 2004,16,1005-1009$.

70. J. Q. Liu, L. Tao, W. R. Yang, D. Li, C. Boyer, R. Wuhrer, F. Braet and T. P. Davis, Langmuir, 2010, 26, 10068-10075.

71. G. Wang, X. Shen, J. Yao and J. Park, Carbon, 2009, 47, 2049-2053.

72. A. V. Murugan, T. Muraliganth and A. Manthiram, Chem. Mat.,

$15 \quad 2009,21,5004-5006$.

73. J. Yan, T. Wei, Z. Fan, W. Qian, M. Zhang, X. Shen and F. Wei, Journal of Power Sources, 2010, 195, 3041-3045.

74. Y. Zhu, M. D. Stoller, W. Cai, A. Velamakanni, R. D. Piner, D. Chen and R. S. Ruoff, ACS Nano, 2010, 4, 1227-1233.

20 75. L. Gomez De Arco, Y. Zhang, C. W. Schlenker, K. Ryu, M. E. Thompson and C. Zhou, ACS Nano, 2010, 4, 2865-2873.

76. S. S. Li, K. H. Tu, C. C. Lin, C. W. Chen and M. Chhowalla, ACS Nano, 2010, 4, 3169-3174.

77. L. Valentini, M. Cardinali, S. B. Bon, D. Bagnis, R. Verdejo, M. A.

25 Lopez-Manchado and J. M. Kenny, J. Mater. Chem., 2010, 20, 9951000.

78. X. Wang, L. Zhi and K. Mullen, Nano Letters, 2007, 8, 323-327.

79. G. Eda, H. E. Unalan, N. Rupesinghe, G. A. J. Amaratunga and M. Chhowalla, Appl. Phys. Lett., 2008, 93, 233502-233503.

30 80. V. P. Verma, S. Das, I. Lahiri and W. Choi, Appl. Phys. Lett., 2010, 96, 203108.

81. P. Simon and Y. Gogotsi, Nat Mater, 2008, 7, 845 - 854.

82. Y. Lee, S. Bae, H. Jang, S. Jang, S.-E. Zhu, S. H. Sim, Y. I. Song, B. H. Hong and J.-H. Ahn, Nano Letters, 2010, 10, 490-493.

35 83. S. Bae, H. Kim, Y. Lee, X. Xu, J.-S. Park, Y. Zheng, J. Balakrishnan, T. Lei, H. Ri Kim, Y. I. Song, Y.-J. Kim, K. S. Kim, B. Ozyilmaz, J.H. Ahn, B. H. Hong and S. Iijima, Nat Nanotechnol, 2010, 5, 574578.

84. H. A. Becerril, J. Mao, Z. Liu, R. M. Stoltenberg, Z. Bao and Y.

40 Chen, ACS Nano, 2008, 2, 463-470.

85. X. Li, G. Zhang, X. Bai, X. Sun, X. Wang, E. Wang and H. Dai, Nat Nano, 2008, 3, 538-542.

86. Y. F. Lian, Y. X. Liu, T. Jiang, J. Shu, H. Q. Lian and M. H. Cao, J. Phys. Chem. C, 2010, 114, 9659-9663.

45 87. R. A. Vaia and J. F. Maguire, Chem. Mat., 2007, 19, 2736-2751. 